\title{
USO DAS TÉCNICAS DE INFRAVERMELHO E DE RESSONÂNCIA MAGNÉTICA NUCLEAR NA CARACTERIZAÇÃO DA REAÇÃO ÁCIDO-BASE DE UM CIMENTO ODONTOLÓGICO EXPERIMENTAL
}

\author{
Marcio José Bertolini* e Maria Aparecida Zaghete \\ Instituto de Química de Araraquara, Universidade Estadual Paulista, R. Francisco Degni, s/n, 14801-970 Araraquara - SP, Brasil \\ Rossano Gimenes \\ Instituto de Ciências Exatas, Universidade Federal de Itajubá, Campus Prof. J. R. Seabra, 37500-000 Itajubá - MG, Brasil
}

Recebido em 8/8/08; aceito em 12/12/08; publicado na web em 28/5/09

\begin{abstract}
USE OF INFRARED AND MAGNETIC NUCLEAR RESONANCE TECHNIQUES IN THE CHARACTERIZATION OF THE ACID-BASE REACTION OF AN EXPERIMENTAL DENTAL CEMENT. Glass ionomer cements (GICs) are products of the acidbase setting reaction between an finely fluoro-alumino silicate glass powder and poly(acrylic acid) in aqueous solution. The sol gel method is an adequate route of preparation of the glasses used to obtain the GICs. The objective of this paper was to compare two powders: a commercial and an experimental and to investigate the structural changes during hardening of the cements by FTIR and Al MAS NMR. These analyses showed that the experimental glass powder reacted with organic acid to form the GICs and it is a promising material to manufacture dental cements.
\end{abstract}

Keywords: cements; polyacrylate; ionomer.

\section{INTRODUÇÃO}

Os cimentos de ionômeros de vidro (CIV) foram desenvolvidos na década de 70 por Wilson e Kent, com os estudos sobre os cimentos de silicatos, onde o ácido fosfórico foi substituído por ácidos orgânicos para diminuir a irritação à polpa dentária. Desse modo, o termo cimento de ionômero de vidro foi utilizado para descrever um composto híbrido formado pela mistura de pó de vidro e ácidos orgânicos. ${ }^{1,2}$ Desde da sua invenção, os CIV vem sendo amplamente estudados para uso em Dentítstica Restauradora.

As pesquisas e os testes que vem sendo realizados com os vidros precursores dos CIV servem para a melhoria das propriedades desses materiais, tais como reatividade, translucidez e propriedades mecânicas, ou seja, tudo contribui para o desenvolvimento e aprimoramento de novas formulações de vidros para serem usados como precursores de cimentos.

As características atrativas dos CIV são boa adesividade ao esmalte e à dentina, valor do coeficiente de expansão térmica linear semelhante ao do dente, efeito anticariogênico e também biocompatibilidade com os tecidos dentários. ${ }^{3-6}$ Segundo levantamentos realizados em países desenvolvidos, há um elevado percentual de profissionais que utilizam esses cimentos odontológicos em procedimentos que vão desde os preventivos, passando por procedimentos curativos tradicionais (como restaurações, bases e forramento de cavidades) até como agentes de cimentação em tratamentos ortodônticos e protéticos.

As composições dos vidros, utilizados como formadores de cimentos, são complexas e variadas. Embora, haja uma variedade de composições, pode-se dizer que essas apresentam alguns constituintes essenciais, tais como sílica $\left(\mathrm{SiO}_{2}\right)$, alumina $\left(\mathrm{Al}_{2} \mathrm{O}_{3}\right)$ e fluoreto, que estão presentes em todas as composições dos vidros comerciais formadores de cimentos. ${ }^{2}$

A maioria desses sistemas vítreos é preparada pelo processo de fusão de mistura de óxidos na faixa de temperatura de 1100 a 1500 ${ }^{0} \mathrm{C}$, dependendo da composição. Mas este processo convencional de síntese de vidros apresenta alguns inconvenientes, tais como dificuldade de se controlar a estequiometria e a volatilização de fluoreto que

*e-mail: marciobert@yahoo.com.br ocorre na faixa de 900 a $1000{ }^{\circ} \mathrm{C}$. Por isso, método químico como o processo sol-gel é usado para preparar vidros contendo fluoreto em temperaturas menores que $1000^{\circ} \mathrm{C}$. Algumas vantagens deste método em relação ao processo convencional de fusão de mistura de óxidos são menor temperatura de processamento, que evita a volatilização de fluoreto; maior homogeneidade e, a possibilidade da obtenção de vidros baseados em sistemas multicomponentes com distribuição de partícula controlada e fase única., ${ }^{7,8}$

A reação ácido/base, responsável pela formação dos CIV, envolve a hidrólise ácida das ligações $\mathrm{Si}-\mathrm{O}-\mathrm{Al}$ da rede vítrea resultando na liberação dos cátions $\mathrm{Ca}^{2+}$ e $\mathrm{Al}^{3+}$ e na formação de ácido ortosilícico, o qual se polimeriza formando sílica gel. Os cátions liberados da rede vítrea $\mathrm{Ca}^{2+} \mathrm{e} \mathrm{Al}^{3+}$ são quelados pelos grupos carboxílicos, presentes na estrutura polimérica do ácido orgânico, formando sais de poliacrilato, responsáveis pela reação de presa inicial da pasta formada pela mistura do pó de vidro com a solução aquosa do ácido orgânico. Dessa maneira, o cimento obtido é constituído de partículas vítreas revestidas por camada de sílica gel presentes em uma matriz formada pelos sais de poliacrilato de cálcio e de alumínio. ${ }^{5-7}$

Em recente artigo, foi demonstrada a preparação e caracterização de um cimento odontológico obtido a partir do sistema vítreo $\mathrm{SiO}_{2}-\mathrm{Al}_{2} \mathrm{O}_{3}-\mathrm{CaO}$ preparado por método químico, ${ }^{9}$ considerando que a inclusão de fluoreto na formulação do pó do vidro precursor dos CIV é importante nas aplicações odontológicas, pois os íons fluoreto liberados por esses cimentos aumentam a resistência contra cáries secundárias. Dessa forma, esse novo artigo reporta a síntese por método químico e a caracterização estrutural por ressonância magnética nuclear (RMN) e infravermelho (FTIR), de um pó experimental baseado no sistema vítreo $\mathrm{SiO}_{2}-\mathrm{Al}_{2} \mathrm{O}_{3}-\mathrm{CaO}-\mathrm{CaF}_{2}$ visando aplicações como precursores de cimentos odontológicos.

\section{PARTE EXPERIMENTAL}

\section{Preparação dos pós pelo método químico}

O processo utilizado para preparar os pós precursores dos cimentos foi o processo sol-gel e os reagentes utilizados foram tetraetilortossilicato (TEOS), nitrato de alumínio, nitrato de cálcio e 
ácido fluorsilício $\left(\mathrm{H}_{2} \mathrm{SiF}_{6}\right)$. Na primeira etapa misturou-se TEOS e etanol sob agitação, a temperatura ambiente por $1 \mathrm{~h}$ (solução A). $\mathrm{Na}$ segunda etapa, as soluções aquosas contendo $\mathrm{Al}^{3+}, \mathrm{Ca}^{2+}$ e o $\mathrm{H}_{2} \mathrm{SiF}_{6}$ foram adicionadas à solução $\mathrm{A}$. As razões etanol/TEOS e água/TEOS usadas nessa etapa foram iguais a 7 .

O sistema permaneceu sob agitação e aquecimento constante $\pm 80^{\circ} \mathrm{C}$ até a obtenção do gel. $\mathrm{O}$ gel obtido foi seco, moído em almofariz de ágata e tratado termicamente a $250{ }^{\circ} \mathrm{C} / 4 \mathrm{~h}$, para eliminação do solvente. Posteriormente, os pós foram calcinados na faixa de temperatura de 600 a $900{ }^{\circ} \mathrm{C}$ por $2 \mathrm{~h}$.

\section{Preparação dos cimentos de ionômero de vidro (CIV)}

Os pós calcinados foram primeiramente moídos em almofariz de ágata e, posteriormente, peneirados para se obter grãos de tamanhos $<25 \mu$ m e então utilizados para preparar os cimentos.

Os CIV foram preparados pela aglutinação do pó ao líquido (solução aquosa de ácido poliacrílico, 45-50\% (m/m)) sobre uma placa de vidro, sendo que a mistura foi feita entre $20 \mathrm{~s}$ a $1 \mathrm{~min}$. Foi usada razão mássica (pó:líquido) igual a 1:1 para os cimentos experimentais e para o CIV comercial foi utilizada a razão de 2,7:1 (recomendada pelo fabricante).

\section{Caracterização dos pós e dos cimentos}

Para determinar a estrutura cristalina dos pós preparados por solgel e calcinados a diferentes temperaturas, fez-se a caracterização por difratometria de raios $\mathrm{X}$ usando radiação $\mathrm{K} \alpha$ do $\mathrm{Cu}$ no equipamento Rint 2000 com ânodo rotatório de $\mathrm{Cu}$, marca Rigaku.

Os espectros de FTIR foram obtidos no espectrofotômetro Nicolet modelo Impact 400. Para a obtenção dos espectros, os pós foram triturados e misturados com $\mathrm{KBr}$ e prensados na forma de pastilhas.

Os espectros de RMN foram feitos em um espectrômetro INOVA 300 Varian. Os espectros de (Magic Angle Spinning) ${ }^{27} \mathrm{Al}-\mathrm{MAS}$ $(78,15 \mathrm{MHz})$ foram obtidos com rotor de Si3N4 com $\pi / 6$ pulsos de $2 \mu$ s e $\mathrm{Al}\left(\mathrm{H}_{2} \mathrm{O}\right) 6^{3+}$ como referência.

\section{RESULTADOS E DISCUSSÃO}

Após o término do processo de calcinação a várias temperaturas, os pós preparados pelo processo sol-gel, foram caracterizados por DRX. Os difratogramas obtidos estão ilustrados na Figura 1.

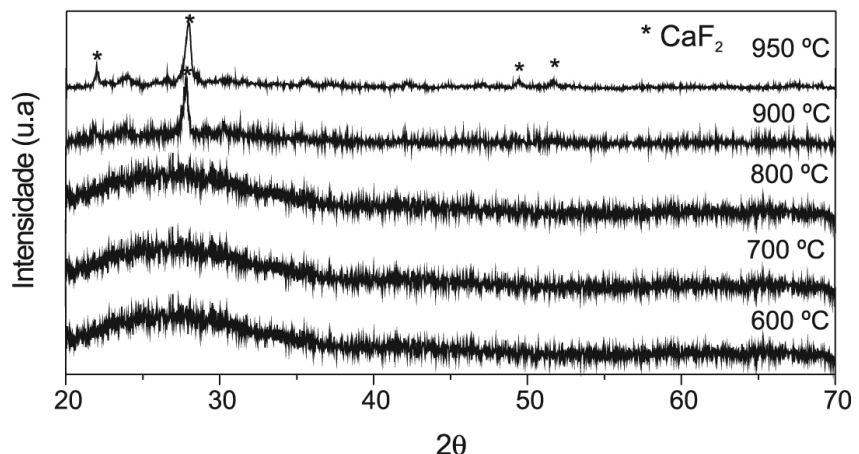

Figura 1. Difratogramas de raios $X$ dos pós calcinados na faixa de temperatura de 600 a $950^{\circ} \mathrm{C}$ por $2 \mathrm{~h}$

Os difratogramas da Figura 1 apresentam um halo típico de materiais amorfos até a temperatura de $800{ }^{\circ} \mathrm{C}$. Acima dessa temperatura, começa a cristalização de uma fase que no difratograma do pó calcinado a $950{ }^{\circ} \mathrm{C}$ aparece mais definida. Essa fase cristalina corresponde à fluorita $\left(\mathrm{CaF}_{2}\right.$ - JPCDF 4-864), a qual tem mostrado também cristalizar em outros preparados pelo método convencional de fusão de mistura de óxidos, inclusive em temperaturas menores que a temperatura de transição vítrea, pelo mecanismo de nucleação de superfície. ${ }^{10}$

Pelos resultados de DRX obtidos pode-se dizer que a partir de $800^{\circ} \mathrm{C}$ tem-se materiais vitro-cerâmicos e abaixo dessa temperatura, materiais amorfos.

As análises de FTIR foram realizadas para verificar a presença das ligações $\mathrm{Si}-\mathrm{O}$ e Al-O-Si, as quais formam a rede vítrea dos pós precursores dos CIV.

Os espectros de FTIR do pó experimental e do pó comercial estão ilustrados na Figura 2.

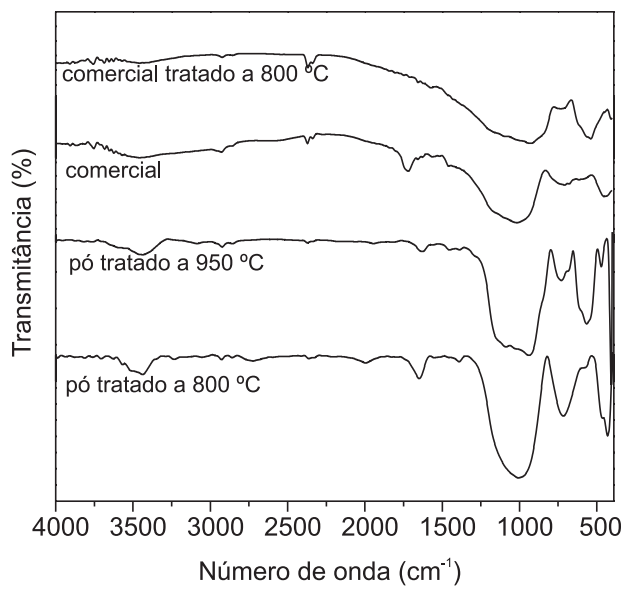

Figura 2. Espectros de FTIR dos pós experimental, calcinado a 800 e a $950^{\circ} \mathrm{C}$ por $2 \mathrm{~h}$, e comercial, sem tratamento térmico e depois calcinado a $800^{\circ} \mathrm{C}$

Em todos os espectros nota-se a presença de bandas referentes aos modos vibracionais da água $v \mathrm{HO}\left(3500 \mathrm{~cm}^{-1}\right), \delta \mathrm{HOH}$ $\left(1600 \mathrm{~cm}^{-1}\right)$ e aos modos referentes às ligações $\mathrm{Si}-\mathrm{O}$ e $\mathrm{Al}-\mathrm{O}-\mathrm{Si}$, tais como $v_{\text {as }}$ SiOSi $\left(1100-1000 \mathrm{~cm}^{-1}\right), v_{\mathrm{s}}$ SiOSi $\left(720-700 \mathrm{~cm}^{-1}\right)$ e $\delta$ AlOSi $\left(460-400 \mathrm{~cm}^{-1}\right)$ que constituem a rede vítrea dos materiais. ${ }^{11}$ Também observa-se uma banda localizada em torno de $600 \mathrm{~cm}^{-1}$ atribuída à presença dos tetraedros de alumínio $\left[\mathrm{AlO}_{4}\right]$ condensados e presentes na rede vítrea. Dessa maneira, pode-se inferir que a rede vítrea dos pós experimentais e do comercial é formada pelos tetraedros $\left[\mathrm{SiO}_{4}\right]$ e $\left[\mathrm{AlO}_{4}\right]$ unidos por ligações do tipo $\mathrm{Si}-\mathrm{O}-\mathrm{Al}$.

É importante enfatizar que a posição das bandas referente ao modo vibracional $v_{\text {as }}$ SiOSi muda dependendo do tipo e do teor de cátions modificadores de rede, tais como $\mathrm{Ca}^{+2}$, porque esses íons atuam na despolimerização da rede vítrea.

No espectro do material comercial não tratado termicamente há uma banda localizada em torno de $1700 \mathrm{~cm}^{-1}$, atribuída ao estiramento da ligação $\mathrm{C}-\mathrm{O}$ e que não está presente no espectro do pó comercial tratado a $800^{\circ} \mathrm{C}$. Isso pode ser explicado levando-se em consideração que o fabricante adiciona aos pós alguns componentes orgânicos, geralmente ácidos como tartárico e maleico, que possibilitam ao dentista uma melhoria na manipulação da pasta, no tempo de trabalho e no tempo de presa inicial dos CIV.

A reação de presa inicial dos CIV experimental foi acompanhada por FTIR (Figura 3), porque as bandas relacionadas com o ácido orgânico, localizadas em torno de 1250 e $1715 \mathrm{~cm}^{-1}$, são progressivamente substituídas por outras bandas atribuídas aos sais de poliacrilato de $\mathrm{Al}$ e Ca formados durante a reação ácido/base entre o pó do vidro e o ácido orgânico. ${ }^{12}$

Como pode ser observado nos espectros dos CIV (Figura 3) após o período de 24 h da manipulação dos cimentos, há bandas referentes 


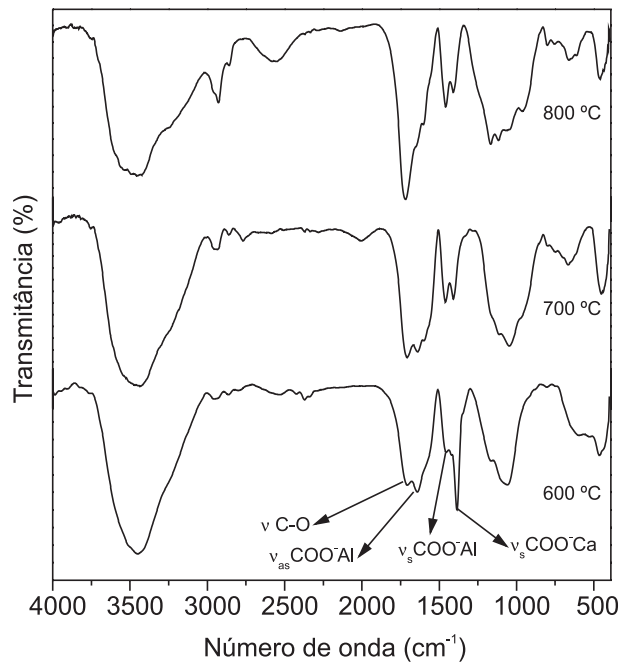

Figura 3. Espectros de FTIR dos CIV experimentais preparados com razão igual a 1:1 após 24 h da manipulação

ao ácido poliacrílico e aos sais de poliacrilato de $\mathrm{Al}$ em torno de 1650 e $1460 \mathrm{~cm}^{-1}$ e de Ca em torno de $1400 \mathrm{e} 1550 \mathrm{~cm}^{-1}$, para todos os cimentos experimentais.

Nos espectros da Figura 3, a banda localizada em torno de 1700 $\mathrm{cm}^{-1}$, referente ao ácido orgânico, é mais intensa para o CIV preparado a partir do pó calcinado a $800^{\circ} \mathrm{C}$. Por outro lado, a intensidade dessa banda é menor para os CIVs preparados a partir dos pós calcinados a 600 e $700{ }^{\circ} \mathrm{C}$, devido ao fato que as as bandas referentes aos sais de poliacrilato de $\mathrm{Al}$ e $\mathrm{Ca}$ se tornaram mais definidas nos espectros desses cimentos.

De modo geral, pode-se considerar que quanto mais rápido as bandas referentes ao ácido poliacrílico forem substituídas pelas bandas dos sais de poliacrilato, menor será o tempo de presa inicial dos CIV, evidenciando que o pó precursor do cimento tem uma estrutura mais vulnerável ao ataque ácido e libera mais facilmente os íons $\mathrm{Al}^{+3} \mathrm{e} \mathrm{Ca}^{+2}$ para formar os respectivos sais de poliacrilato. Com relação a essa observação, nota-se que a banda referente ao sal de poliacrilato de cálcio também está mais intensa no espectro do CIV preparado a partir do pó calcinado a $600^{\circ} \mathrm{C}$, indicando que esse cimento possui um tempo de presa inicial menor em relação aos demais cimentos experimentais preparados a partir dos pós calcinados a 700 e $800^{\circ} \mathrm{C}$.

Os pós precursores dos CIV comercial e experimental, calcinados a $600{ }^{\circ} \mathrm{C}$, assim como os CIV preparados a partir desses pós foram caracterizados por RMN Al MAS para verificar se houve alguma alteração do ambiente dos átomos de $\mathrm{Al}$ na estrutura dos vidros, após a reação com o ácido poliacrílico. As Figuras 4 e 5 ilustram os espectros de RMN Al MAS dos pós e dos CIV comercial e experimental.

De acordo com a literatura, quando os átomos de $\mathrm{Al}$ estão tetraedricamente coordenados $\left(\mathrm{Al}^{\mathrm{IV}}\right)$ um pico referente ao deslocamento químico na faixa de 55 a 80 ppm é observado no espectro. Se a rede vítrea tiver também átomos de $\mathrm{Al}$ octaedricamente coordenados $\left(\mathrm{Al}^{\mathrm{VI}}\right)$, um segundo pico na faixa de -10 a 10 ppm também aparece no espectro. ${ }^{13,14}$

Nos espectros de RMN Al (Figura 4) os picos localizados em 54 e 2 ppm (para o pó experimental) e em 54,2 e -5 ppm (para o pó comercial) indicam que os átomos de $\mathrm{Al}$ também estão tetraedrica e octaedricamente, ${ }^{13,14}$ coordenados na rede vítrea desses materiais.

Analisando os espectros da Figura 5, nota-se que os átomos de Al estão tetraedrica e octaedricamente coordenados após a reação com o ácido poliacrílico, A representação esquemática da reacão ácido/ base está ilustrada na Figura 6.

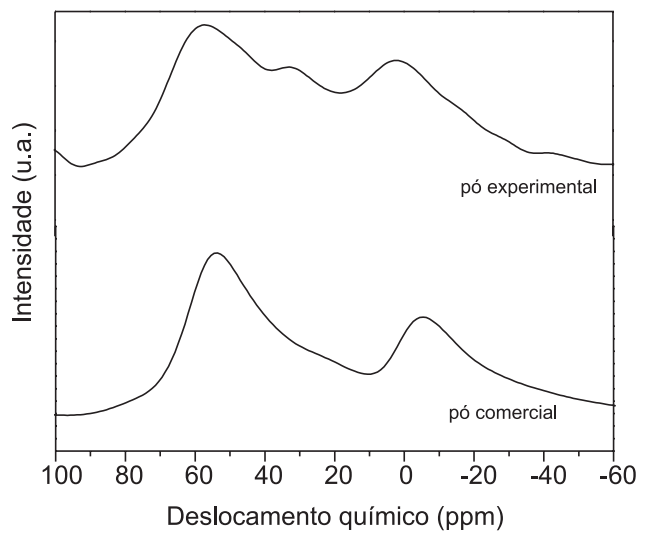

Figura 4. Espectros de RMN de ${ }^{27} \mathrm{Al}$ MAS dos pós comercial e experimental calcinados a $600^{\circ} \mathrm{C} / 2 \mathrm{~h}$

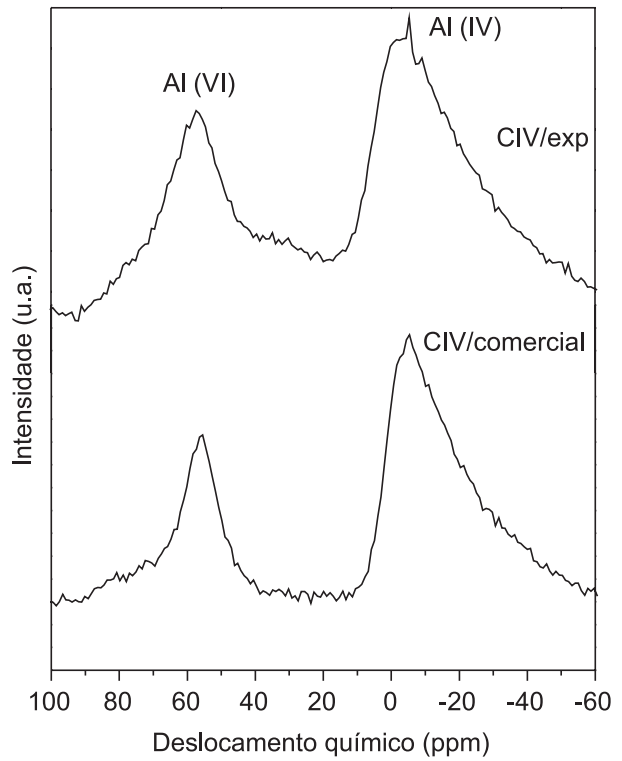

Figura 5. Espectros de RMN de ${ }^{27} \mathrm{Al}$ MAS dos CIV comercial e experimental após 24 h da manipulação

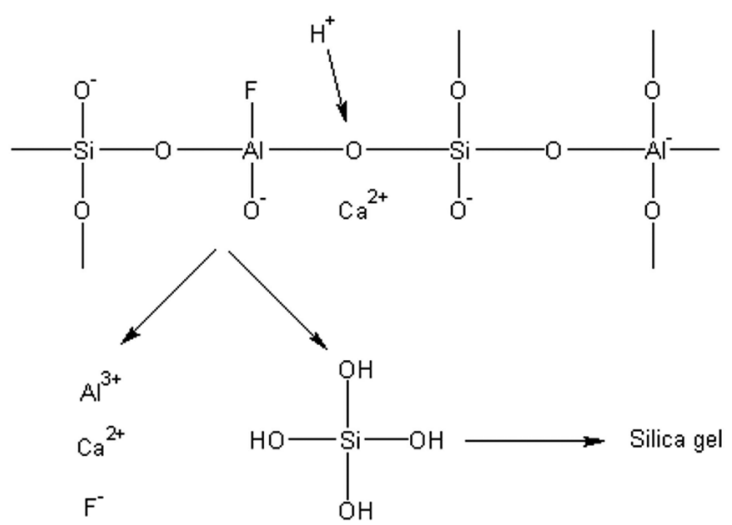

Figura 6. Representação do ataque ácido à rede vítrea formada pelos tetraedros $\mathrm{SiO}_{4}{\mathrm{e} \mathrm{AlO}_{4}}$

Como ilustrado na Figura 6, quando ocorre o ataque ácido os íons $\mathrm{Al}^{3+}, \mathrm{Ca}^{2+}$ e $\mathrm{F}^{-}$vão sendo liberados da rede vítrea, a qual vai sendo rompida e forma-se uma camada rica em ácido ortosilícico que se polimeriza, resultando em uma camada de sílica gel ao redor das partículas vítreas. 
Observa-se que ambos os CIV apresentam espectros semelhantes (Figura 5) e mais resolvidos que os apresentados na Figura 4. Os picos referentes aos átomos de $\mathrm{Al}$ (VI) estão mais definidos e aumentaram de intensidade, indicando que houve liberação dos íons $\mathrm{Al}^{3+}$ da rede vítrea, e que esses foram quelados pelos grupos $\mathrm{COO}^{-}$do ácido poliacrílico formando o sal poliacrilato de alumínio. ${ }^{15,16}$ Confirmando, assim, os dados obtidos por FTIR, onde também foi observada a formação de poliacrilato de aluminio e de cálcio De acordo com Culbertson, ${ }^{7}$ algumas das possíveis estruturas moleculares desses sais de poliacrilato formados pela reação ácido/base na preparação dos CIV são ilustradas pela Figura 7. Nota-se que íons $\mathrm{Ca}^{2+}{\mathrm{e}{ }^{3+}}^{3+}$ estão ligados aos grupos $\mathrm{COO}^{-}$e às moléculas de água. Embora, o $\mathrm{Al}^{3+}$ seja um cátion trivalente, em meio aquoso, ele possui um número de coordenação igual a 6.
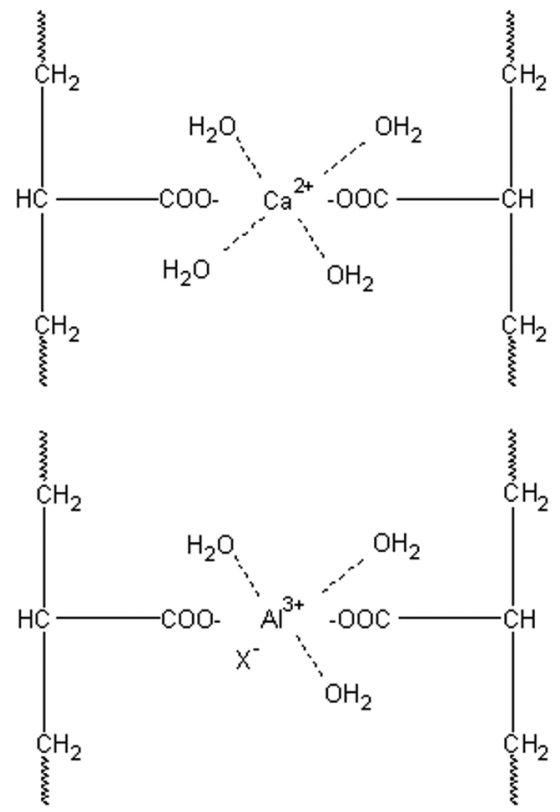

Figura 7. Representação das possíveis estruturas dos sais de poliacrilatos formados durante a reação de formação dos cimentos, onde $\mathrm{X}=\mathrm{F}^{-1} \mathrm{ou} \mathrm{OH}^{-1}$
Esses resultados obtidos pelas técnicas de FTIR e RMN comprovam a reatividade do pó experimental e que este pode ser utilizado na preparação de cimentos odontológicos.

\section{CONCLUSÃO}

O pó experimental preparado pelo processo sol-gel apresenta característica estrutural semelhante ao pó comercial.

As técnicas de FTIR e RMN comprovaram que houve a formação de sais poliacrilato de $\mathrm{Ca}$ e de $\mathrm{Al}$, o que demonstra que o pó experimental é reativo frente ao ácido orgânico e pode ser usado na obtenção de cimentos odontológicos.

\section{REFERÊNCIAS}

1. Abouch, Y. E. Y.; Torabzadeh, H.; J. Can. Dent Assoc. 1998, 64, 561.

2. Costa, C. A. S.; Girob, E. M. A.; Nascimento, A. B. L.; Teixeira, H. M.; Hebling, J.; Dent. Mater. 2003, 19, 739.

3. Mathis, R. S.; Ferrance, J. L.; Dent. Mater. 1989, 5, 355.

4. Herrera, M.; Castilho, A.; Bravo, M.; Liébana, J.; Carrión, P.; Operative Dentistry 2000, 25, 265.

5. Loyola-Rodriguez, J. P.; Garcia-Godoy, F.; Lindquist, R.; Pediatric Dentistry 1994, 16, 346.

6. Nicholson, J. W.; Czarnecka, B.; J. Oral Rehabil. 2004, 31, 665.

7. Culbertson, B. M.; Prog. Polym. Sci. 2001, 26, 577.

8. Taira, M.; Yamaki, M.; J. Mater. Sci.: Mater. Med. 1995, 6, 197.

9. Bertolini, M. J.; Zaghete, M. A.; Gimenes, R.; Paiva-Santos, C. O. P.; Palma-Dibb, R. G.; Quim. Nova 2005, 28, 813.

10. Hill, R. G.; Goat, C.; Wood, D.; J. Am. Ceram. Soc. 1992, 75, 778.

11. Hwa, L. G.; Hwang, S. L.; Liu, L. C.; J. Non-Cryst. Solids 1998, 238, 193.

12. Young, A. M.; Biomaterials 2002, 23, 3289.

13. Schneider, J.; Mastelaro, V. R.; PanepuccI, H.; Zanotto, E. D.; J. NonCryst. Solids 2000, 273, 8.

14. Matsuya, S.; Stamboulis, A.; Hill, R. G.; Law, R. V.; J. Non-Cryst. Solids 2007, 353, 237.

15. Nourmohammadi, J.; Salarian, R.; Solati-Hashjin, M.; Moztarzadeh, F.; Ceram. Int. 2007, 33, 557.

16. Matsuya, S.; Stamboulis, A.; Hill, R. G.; Law, R. V.; Udoh, K.; Nakagawa, M.; Matsuya, Y.; J. Dentistry 2006, 34, 574. 